\title{
Prevalência da fragilidade entre os pacientes com doença renal crônica em tratamento conservador e em diálise
}

\author{
Prevalence of frailty in patients in chronic kidney disease on \\ conservative treatment and on dialysis
}

\begin{abstract}
Autores
Henrique Novais Mansur ${ }^{1}$ Vinícius de Oliveira Damasceno $^{1}$

Marcus Gomes Bastos²

'Universidade Salgado de Oliveira.

${ }^{2}$ Núcleo Interdisciplinar de Estudo, Pesquisa e Tratamento em Nefrologia (NIEPEN); Fundação IMEPEN.
\end{abstract}

Data de submissão: 09/11/2011 Data de aprovação: 16/01/2012

\section{Correspondência para:} Henrique Novais Mansur Rua Wolfgang Amadeus Mozart, 131

Cond. São Lucas I São Pedro

Juiz de Fora - MG - Brasil CEP 36036-631

E-mail: hnmansur@gmail. com

Suporte financeiro: Fundação Instituto Mineiro de Estudos e Pesquisas em Nefrologia - Fundação IMEPEN

O referido estudo foi realizado na Fundação Instituto Mineiro de Ensino, Pesquisa eTratamento em Nefrologia.

Os autores declaram a inexistência de conflitos de interesse.

\section{Resumo}

Introdução: Fragilidade é um estado de vulnerabilidade fisiológica do paciente que se associa ao maior número de hospitalização e óbito. Objetivos: Avaliar a prevalência de fragilidade e os fatores a ela associados em pacientes com doença renal crônica (DRC) em tratamento conservador (TC), hemodiálise (HD) e diálise peritoneal (DP). Métodos: A fragilidade foi avaliada em 146 pacientes (86 em TC, 37 em HD e 23 em DP) e caracterizada como fraqueza muscular e exaustão - pelos domínios do aspecto físico e da vitalidade, respectivamente, avaliados pelo questionário SF-36 de qualidade de vida; como inatividade física caso a resposta fosse "nunca" ou "quase nunca" - quando questionado sobre a prática de atividade física; e como perda de peso não intencional ( $\geq 4,5 \mathrm{~kg}$ por ano). Os pacientes foram divididos em três grupos: não frágeis $(\mathrm{NF})$, pré-frágeis $(\mathrm{PF})$ e frágeis $(\mathrm{F})$. As variáveis demográficas, clínicas e laboratoriais foram extraídas dos prontuários dos pacientes. Resultados: A fragilidade foi caracterizada em $36 \%$ dos pacientes em TC, $37,8 \%$ em HD e 47,8\% em DP. Foi diagnosticada em $36,8 \%$ dos pacientes com idade entre 20 e 40 anos e 40,3\% daqueles entre 41 e 60 anos. A fragilidade associou-se significativamente ao uso de vitamina $\mathrm{D}(\mathrm{r}=0.16 ; \mathrm{p}=0.03)$, hemoglobina $(\mathrm{r}=-0.14 ; \mathrm{p}=-0.02)$ e paratormônio intacto $(r=0.16 ; p=0.03)$. Conclusões: A fragilidade é frequente entre os pacientes com DRC em tratamento conservador e dialítico, mesmo naqueles não idosos. Nos pacientes estudados, o fenótipo de fragilidade se associou com o não uso de vitamina $\mathrm{D}$, menores níveis séricos de hemoglobina e níveis mais elevados de paratormônio.

Palavras-chave: Diálise Renal. Diálise peritoneal. Insuficiência renal crônica.

\section{Abstract}

Introduction: Frailty is a physiological vulnerability status of the patient which is associated with the increased number of hospitalization and death. Objectives: To evaluate the prevalence of frailty and its associated factors in patients with chronic kidney disease (CKD) on conservative treatment (CT), hemodialysis (HD) and peritoneal dialysis (PD). Methods: Frailty was assessed in 146 patients (86 CT, $37 \mathrm{HD}$ and $23 \mathrm{PD}$ ) and characterized as muscle weakness and exhaustion - by the physical aspect and vitality domains, respectively, evaluated in the SF-36 quality of life instrument; physical inactivity - if he or she answered "never" or "hardly ever" when asked about physical activity; and as unintentional weight loss $(\geq 4.5 \mathrm{~kg}$ per year). Patients were divided into three groups: non-fragile (NF), pre-fragile (PF) and fragile $(\mathrm{F})$. The demographic, clinical and laboratory data were extracted from patient charts. Results: Frailty was diagnosed in $36 \%$ of patients on CT, $37.8 \%$ in $\mathrm{HD}$ and $47.8 \%$ in PD. It was characterized in $36.8 \%$ of patients aged between 20 and 40 years and $40.3 \%$ of those between 41 and 60 years. Frailty was significantly associated with the use of vitamin $\mathrm{D}(\mathrm{r}=0.16 ; \mathrm{p}=0.03)$, hemoglobin $(r=-0.14 ; \mathrm{p}=-0.02)$ and intact parathyroid hormone $(\mathrm{r}=0.16 ; \mathrm{p}=0.03)$. Conclusions: Frailty is common among patients with CKD on conservative treatment and dialysis, even in those who are not elderly. In the patients studied, the phenotype of frailty was associated with no usage of vitamin $\mathrm{D}$, lower serum levels of hemoglobin and higher levels of parathyroid hormone.

Keywords: Renal dialysis. Peritoneal dialysis. Renal insufficiency, chronic. 


\section{INTRODUÇÃO}

A doença renal crônica (DRC) já é considerada um dos maiores problemas de saúde pública da atualidade em todo o mundo. ${ }^{1}$ Uma grande dificuldade no manejo da DRC deve-se ao seu curso, geralmente silencioso, nos estágios iniciais da doença, o que dificulta seu diagnóstico precoce. Outro aspecto relevante é a complexidade das alterações decorrentes da diminuição progressiva da taxa de filtração glomerular (TFG) que determina complicações, propicia as comorbidades (principalmente as cardiovasculares), aumenta a mortalidade precoce e a falência funcional renal. Os pacientes com DRC também apresentam redução da qualidade de vida e da cognição, diminuição da atividade física, perda da massa muscular e alta prevalência de sintomas como fadiga, náuseas e anorexia. ${ }^{2,3}$ Com o início da terapia dialítica, é frequente observar piora do status funcional do paciente, condição que se associa com risco aumentado de hospitalização e óbito. ${ }^{4,5}$ Contudo, até o momento, poucos estudos têm explorado a capacidade funcional do paciente no contexto da DRC.

A fragilidade é um estado clínico de vulnerabilidade, comum aos idosos, que prejudica a capacidade de o organismo responder aos agentes estressores, reduzindo as reservas fisiológicas e prejudicando a habilidade do organismo em manter a homeostase. ${ }^{6}$ Caracteriza-se pela perda de peso, de força e de equilíbrio, além de exaustão, de baixa capacidade de exercício e de velocidade de caminhada. Essas alterações aumentam o risco de incapacidade, institucionalização e hospitalização. ${ }^{7}$

A ocorrência frequente de anemia, inflamação, dislipidemia, distúrbio do metabolismo ósseo e mineral, acidose metabólica, desnutrição, disfunção muscular e doenças cardiovasculares, frequentes nos pacientes com DRC, são manifestações clínicas que favorecem a ocorrência do "fenótipo de fragilidade". ${ }^{8}$ Por exemplo, numa análise em 5.888 participantes adultos não institucionalizados e com idade $\geq 65$ anos, a prevalência de fragilidade foi maior nos portadores de DRC $(15 \%)$ do que nos indivíduos sem a doença $(6 \%) .{ }^{8}$ Entre os pacientes em tratamento dialítico, a prevalência de fragilidade chega a atingir um total de $67,7 \%$, e a síndrome frágil foi também caracterizada em portadores de DRC não idosos. Comparados aos pacientes não frágeis, aqueles submetidos à diálise e com o fenótipo de fragilidade apresentam maior risco de óbito e hospitalização. ${ }^{9} \mathrm{O}$ construto de fragilidade pode ser observado em todos os estágios da DRC. É conhecido que ser portador de doença renal aumenta muito as chances de fragilidade quando comparamos os indivíduos com função renal normal ou portadores de outras doenças crônicas (por exemplo, diabetes, doença pulmonar obstrutiva crônica, câncer e artrite reumatoide)..$^{10}$

A alta prevalência de fragilidade e a associação com desfechos adversos justificam a investigação em pacientes com DRC, objetivando a preservação da autonomia, da qualidade de vida e da diminuição da mortalidade. O objetivo do nosso estudo foi avaliar a prevalência de fragilidade e seus fatores associados em pacientes com DRC em tratamento conservador e dialítico.

\section{Métodos}

\section{PACIENTES}

Foram estudados 146 pacientes, 86 em tratamento conservador (TC), 37 em hemodiálise (HD) e 23 em diálise peritoneal (DP) do Programa de DRC do Núcleo Interdisciplinar de Estudo, Pesquisa e Tratamento em Nefrologia - NIEPEN da Universidade Federal de Juiz de Fora. Foram incluídos todos os pacientes que aceitaram participar voluntariamente da pesquisa, e não incluídos aqueles previamente transplantados, com amputações de membro inferior ou com grande dificuldade de compreensão das questões da entrevista.

$\mathrm{O}$ tratamento hemodialítico era realizado três vezes por semana, por quatro horas, por meio de fístula artério-venosa. Todos os pacientes em DP eram tratados com máquina cicladora. Os pacientes em TC apresentavam DRC nos estágios 2 a 5.

O projeto foi aprovado pelo Comitê de Ética e Pesquisa do Hospital Universitário de Juiz de Fora.

\section{DIAGNÓSTICO DE FRAGILIDADE}

O diagnóstico da síndrome da fragilidade baseou-se na proposta de Johansen et al..$^{9} \mathrm{e}$ foi realizado mediante entrevista presencial ou contato telefônico. Os pacientes com pontuação zero foram considerados não frágeis (NF); com 1 ou 2 pontos, pré-frágeis (PF); e 3 ou mais critérios, frágeis $(\mathrm{F})$ (Tabela 1).

\section{DADOS CLÍNICOS E LABORATORIAIS}

Os dados relativos a idade, sexo, fenótipo negro e não negro, peso, estatura, índice de massa corporal (IMC), etiologia da DRC e comorbidades, medicamentos em uso (colecalciferol, calcitriol, agente estimulador da eritropoiese ou AEE, sacarato de hidróxido de ferro), dados laboratoriais (creatinina sérica, hemoglobina, albumina, cálcio, fósforo, PTH molécula intacta - PTH-i, hormônio estimulador da 


\section{Tabela 1 DeFINIÇÃo DE FRAGILIDADE ${ }^{9}$}

\begin{tabular}{ll} 
Componentes do fenótipo de fragilidade & Método de avaliação \\
\hline Fraqueza muscular* & $<75$ pontos no domínio Aspecto Físico do SF-36\& \\
Exaustão** & $<55$ pontos no domínio Vitalidade do SF-36\& \\
Inatividade física** $^{*}$ & Resposta "quase nunca ou nunca" à pergunta: "Com qual frequência \\
& você pratica atividade física no seu tempo livre? \\
Perda de peso não intencional &
\end{tabular}

*A ocorrência de fraqueza muscular corresponde a dois pontos e os componentes **exaustão, **inatividade física e **Perda de peso não intencional equivalem a um ponto cada.

\&Medical Outcomes Study 36-item-short-form health survey.

tireóide ultrassensível - TSH-us) e o Kt/V dos pacientes em tratamento hemodialítico foram obtidos dos prontuários dos participantes quando da avaliação da fragilidade. A TFG foi estimada a partir da creatinina sérica por meio da fórmula do estudo Modification of Diet in Renal Disease (MDRD) ${ }^{11}$, e a DRC, estagiada segundo o critério do K/DOQI da National Kidney Foundation americana. ${ }^{12}$

\section{AnÁlise estatística}

As características dos pacientes, incluindo dados demográficos, clínicos e laboratoriais, são apresentadas como média \pm DP ou número de observações (percentagem do total) para as variáveis normais e em mediana para as variáveis não normais por meio do teste de normalidade de Kolmogorov-Smirnov. A comparação entre os grupos foi realizada por ANOVA one-way com análise post hoc de Tukey.

Foi realizado o teste qui-quadrado para identificar as variáveis categóricas associadas estatisticamente com a fragilidade e a correlação de postos Tau b de Kendall para medir a associação entre variáveis identificadas e o construto fragilidade.

O nível de significância adotado foi de $\mathrm{p}<0,05$. Os dados foram analisados pelo software SPSS, versão 17.0 (SPSS Inc, Chicago, IL).

\section{Resultados}

Como demonstrado na Tabela 2, as características dos pacientes que compuseram os grupos de tratamento não foram diferentes quanto a idade, sexo e raça. A prevalência de fragilidade foi ligeiramente inferior entre as mulheres e nos indivíduos de fenótipo negro. Porém, o IMC entre os grupos foi diferente, sendo o grupo HD o único que apresentou índices normais.

Não houve diferença entre os grupos quanto à etiologia da DRC, à ocorrência de comorbidades e ao uso de AEE. O tratamento com vitamina $\mathrm{D}$ nas formas ativa e não ativa foi diferente entre os grupos, assim como os níveis laboratoriais de creatinina, albumina, hemoglobina, cálcio, fósforo, PTH-i e TSH-us (Tabela 2).

A Figura 1 mostra que o fenótipo de fragilidade foi diagnosticado em $38,4 \%$ dos pacientes com DRC, e a prevalência não foi diferente se os pacientes estavam em TC $(36 \%)$, HD $(37,8)$ ou DP $(47,8 \%)$ ( $p>0,05)$. Também pudemos observar que a síndrome frágil foi diagnosticada em pacientes não idosos em todas as modalidades de tratamento.

A presença de um estado pré-frágil, ou seja, a ocorrência de uma ou duas variáveis da síndrome frágil foi observada em 39,7\% dos pacientes e também não se mostrou estatisticamente diferente entre os pacientes em TC $(39,5 \%)$, HD $(40,5 \%)$ e DP $(39,1 \%)$ (dados não apresentados).

A frequência das anormalidades que compõem o fenótipo da fragilidade é apresentada na Tabela 3. Entre os componentes da síndrome frágil, a inatividade física $(\mathrm{p}=0,003)$ e a perda não intencional de peso $(\mathrm{p}=0,000)$ foram as variáveis com frequências estatisticamente diferentes entre os grupos de tratamento.

A Tabela 4 mostra as características demográficas, clínicas e laboratoriais que se associaram com a ocorrência da síndrome frágil. A fragilidade se correlaciona negativamente com o não uso de vitamina $\mathrm{D}$ e o nível sanguíneo de hemoglobina e positivamente com o nível sérico de PTH-i.

\section{Dıscussão}

O fenótipo de fragilidade tem sido associado a desfechos adversos entre os indivíduos idosos na população geral, mas a prevalência e o significado da sua ocorrência entre os pacientes com DRC ainda são pouco estudados. 
Tabela 2 DESCRIÇÃo dOS PARÂMETROS DEMOGRÁFICOS, CLÍNICOS E BIOQUíMICOS DOS PARTICIPANTES DO ESTUDO

\begin{tabular}{|c|c|c|c|c|c|}
\hline Variáveis & $\begin{array}{c}\text { Total } \\
(n=146)\end{array}$ & $\begin{array}{c}\text { Pred } \\
(n=86)\end{array}$ & $\begin{array}{c}\text { HD } \\
(n=37)\end{array}$ & $\begin{array}{c}D P \\
(n=23)\end{array}$ & $\mathrm{p}$ \\
\hline Idade (anos) & $57,7 \pm 13,1$ & $59,9 \pm 12,5$ & $55,3 \pm 11,9$ & $53,5 \pm 15,7$ & NS \\
\hline Sexo feminino, $n(\%)$ & $72(49,3)$ & $37(43)$ & $20(54,1)$ & $15(65,2)$ & NS \\
\hline Fenótipo negro, n(\%) & $56(38,4)$ & $49(57)$ & $20(54,1)$ & $6(26,1)$ & NS \\
\hline$I M C^{€}\left(\mathrm{~kg} / \mathrm{m}^{2}\right)$, média $\pm \mathrm{DP}$ & $25,9 \pm 5,0$ & $27,3 \pm 4,8$ & $23,2 \pm 3,9$ & $25 \pm 5,2$ & 0 \\
\hline Tabagismo, n (\%) & $16(11)$ & $12(14)$ & $4(10,8)$ & $0(0)$ & NS \\
\hline Estágio da DRC, n (\%) & & & & & $0,000^{*}$ \\
\hline 2 & $3(2,1)$ & $3(3,5)$ & $0(0)$ & $0(0)$ & \\
\hline 3 & $37(25,3)$ & $37(43)$ & $0(0)$ & $0(0)$ & \\
\hline 4 & $35(24)$ & $35(40,7)$ & $0(0)$ & $0(0)$ & \\
\hline 5 & $71(48,6)$ & $11(12,8)$ & $37(100)$ & $23(100)$ & \\
\hline Etiologia da DRC, n (\%) & & & & & NS \\
\hline Hipertensão arterial (HA) & $24(16,4)$ & $18(20,9)$ & $3(8,1)$ & $3(13)$ & \\
\hline Diabetes mellitus (DM) & $24(16,4)$ & $13(15,1)$ & $4(10,8)$ & $7(30,4)$ & \\
\hline Glomerulonefrite & $37(25,3)$ & $15(17,4)$ & $15(40,5)$ & $7(30,4)$ & \\
\hline Indeterminada/outras & $61(41,9)$ & $40(46,6)$ & $15(40,5)$ & $6(26,2)$ & \\
\hline Comorbidades, n (\%) & & & & & NS \\
\hline Ausente & $7(4,8)$ & $6(7)$ & $1(3,7)$ & $0(0)$ & \\
\hline $\mathrm{HA}$ & $84(57,5)$ & $35(24)$ & $32(86,4)$ & $17(53,1)$ & \\
\hline DM & $10(6,8)$ & $10(6,8)$ & $0(0)$ & $0(0)$ & \\
\hline Outra & $87(59,5)$ & $33(22,6)$ & $24(64,8)$ & $19(82,6)$ & \\
\hline \multicolumn{6}{|l|}{ Tratamento, n (\%) } \\
\hline$A E E^{f}$ & $69(47,3)$ & $15(17,4)$ & $35(94,6)$ & $19(82,6)$ & NS \\
\hline Vitamina $D^{\ell}$ & $31(21,2)$ & $12(14)$ & $24(37,1)$ & $5(21,7)$ & 0,000 \\
\hline Ferro Venoso ${ }^{\mathrm{e}}$ & $31(21,2)$ & $3(3,5)$ & $28(75,7)$ & $23(100)$ & 0,000 \\
\hline Creatinina $(\mathrm{mg} / \mathrm{dL})$, média $\pm \mathrm{DP}$ & $7,1 \pm 5,4$ & $2,7 \pm 3,1$ & $11,4 \pm 3,4$ & $10,9 \pm 5,3$ & $0,000 * ¥$ \\
\hline PTH-i (pg/mL), média \pm DP & $486 \pm 484,3$ & $240,2 \pm 237,1$ & $646,9 \pm 546,6$ & $763 \pm 530,3$ & $0,000^{* *}$ \\
\hline Hemoglobina $(\mathrm{g} / \mathrm{dL})$, média $\pm \mathrm{DP}$ & $12,2 \pm 2,2$ & $12,9 \pm 2,0$ & $11,2 \pm 2,2$ & $11,3 \pm 2,0$ & $0,000^{* *}$ \\
\hline Albumina (g/dL), média \pm DP & $3,9 \pm 0,7$ & $4,2 \pm 0,3$ & $3,9 \pm 0,8$ & $3,4 \pm 0,2$ & $0,000 * *$ \\
\hline Cálcio (mg/dL), média \pm DP & $9,4 \pm 1,0$ & $9,7 \pm 1,0$ & $9,0 \pm 0,9$ & $9,3 \pm 0,8$ & $0,001 *$ \\
\hline Fósforo (mg/dL), média \pm DP & $5,1 \pm 1,9$ & $3,9 \pm 0,9$ & $6,4 \pm 1,9$ & $7 \pm 1,5$ & $0,000^{* *}$ \\
\hline Ca $\times P^{\partial}(\mathrm{mg} / \mathrm{dL})$, média $\pm \mathrm{DP}$ & $47,7 \pm 19,4$ & $36,7 \pm 11,2$ & $58,8 \pm 19,8$ & $66 \pm 16,5$ & $0,000 * *$ \\
\hline $\operatorname{TSHus}^{\Sigma}(U \mathrm{I} / \mathrm{mL})$, média $\pm \mathrm{DP}$ & $3,1 \pm 2,9$ & $2,4 \pm 2,2$ & $3,4 \pm 3,4$ & $4,9 \pm 2,6$ & $0,007^{¥}$ \\
\hline $\mathrm{Kt} / \mathrm{N}$, média $\pm \mathrm{DP}$ & $0 \pm 0$ & $0 \pm 0$ & $1,4 \pm 0,3$ & $0 \pm 0$ & \\
\hline
\end{tabular}

\&TC : Tratamento conservador; ${ }^{\theta H D}$ : Hemodiálise; ${ }^{\circ D P}$ : Diálise peritoneal; $€$ IMC: índice de massa corporal; ${ }^{\circledR} A E E$ : Agente estimulador da

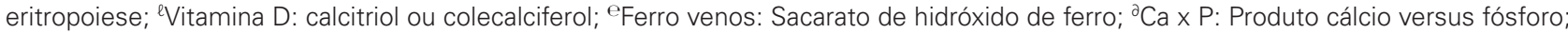
ITSH-us : hormônio estimulador da tireoide ultrassensível; NS = não significativo; ${ }^{*}$ Comparação entre TC e HD, ${ }^{*}$ Comparação entre TC e DP; ${ }^{\S}$ Comparação entre HD e DP; Kt/N não realizado nos pacientes em DP; DRC: doença renal crônica; PTH-i: PTH: molécula intacta.

O termo "fragilidade" tem sido utilizado para caracterizar um grupo de adultos idosos mais fracos e mais vulneráveis. Os resultados de estudos recentes têm ajudado a melhor definir as características clínicas e fisiológicas da fragilidade e destacar a vulnerabilidade do idoso frágil a desfechos adversos. A palavra fragilidade é frequentemente empregada na geriatria clínica e descreve a combinação de fraqueza muscular, fragilidade óssea, índice de massa corporal muito diminuído, susceptibilidade a quedas, vulnerabilidade à trauma e à infecção, risco aumentado para ocorrência de delírio, instabilidade da pressão arterial 
Tabela 3 OCORRÊNCIA DOS COMPONENTES DE FRAGILIDADE ENTRE OS GRUPOS NÃO FRÁGIL, PRÉ-FRÁGIL E FRÁGIL

\begin{tabular}{lcccc} 
Componentes da fragilidade & Não frágil & Pré-frágil & Frágil & Valor p \\
\hline Fraqueza (<75 pontos no SF-36) & $0 \%$ & $11,5 \%$ & $100 \%$ & $0,000^{1,2}$ \\
Exaustão (<55 pontos no SF-36) & $0 \%$ & $6,6 \%$ & $46,6 \%$ & $0,000^{1,2}$ \\
Inatividade Física & $0 \%$ & $83,6 \%$ & $86,2 \%$ & $0,000^{1,3}$ \\
Perda de peso & $0 \%$ & $8,2 \%$ & $20,7 \%$ & $0,000^{1,2,3}$ \\
\hline
\end{tabular}

O componente fraqueza equivale a dois pontos se a pontuação no SF-36 for inferior a 75 pontos. O componente exaustão equivale a um ponto se a pontuação for inferior a 55 no SF-36. ${ }^{1}$ Comparação entre frágil e não frágil. ${ }^{2}$ Comparação entre frágil e pré-fragil. ${ }^{3}$ Pré-frágil e não frágil.

Figura 1. Prevalência da fragilidade na doença renal crônica de acordo com o grupo de tratamento e faixa etária.

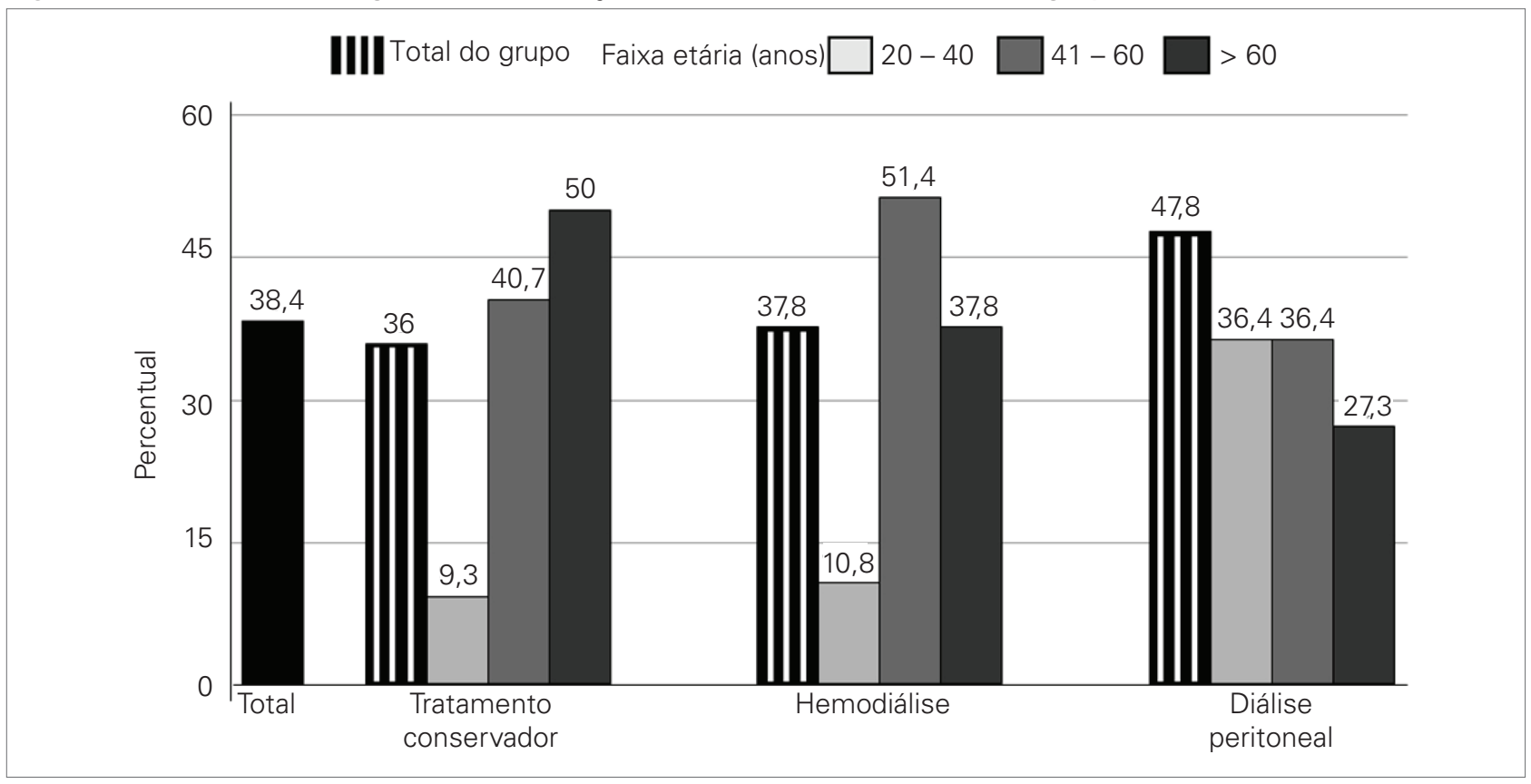

e limitação acentuada das capacidades físicas. Fried et al. ${ }^{6}$ definiram a fragilidade como uma síndrome que engloba traços clínicos associados ao processo de envelhecimento, como fraqueza, fadiga, perda de peso, desequilíbrio, baixo nível de atividade física, perda de peso, atividade e processamento motor mais lentos, exclusão social, alterações cognitivas leves e vulnerabilidade aumentada a estressores.

A associação entre a queda da TFG ou necessidade de TRS com a prevalência de fragilidade e a redução das capacidades físicas e funcionais têm recebido especial atenção nos últimos anos. Shlipak et al. ${ }^{8}$ avaliaram a prevalência de fragilidade numa coorte de pacientes idosos (idade $\geq 65$ anos) e observaram uma taxa geral de $15 \%$ entre os pacientes que apresentavam DRC. As chances de fragilidade foram inversamente relacionadas à TFG, sendo que um quinto dos participantes com menos que $40 \mathrm{~mL} / \mathrm{min} / 1,73 \mathrm{~m}^{2}$ foram caracterizados como frágeis. Prevalência de fragilidade semelhante foi descrita por Wilhelm-Leen et al. ${ }^{10}$ entre

\begin{tabular}{lcc} 
Tabela 4 & $\begin{array}{l}\text { CoRRELAÇÃO ENTRE FRAGILIDADE E } \\
\text { VARIÁVEIS DO ESTUDO }\end{array}$ \\
Variáveis & Valor $r$ & Valor $\mathrm{p}$ \\
\hline Idade &, 052 & 0,429 \\
Raça &, 099 & 0,209 \\
Estágio da DRC &, 116 & 0,118 \\
IMC &, 063 & 0,333 \\
Tabagismo &, 059 & 0,456 \\
Etilogia da DRC &,- 114 & 0,108 \\
Comorbidades &, 077 & 0,476 \\
AEE &, 050 & 0,527 \\
Ferro venoso &,- 046 & 0,562 \\
Vitamina D &,- 167 & $0,031^{*}$ \\
Hemoglobina &,- 147 & $0,028^{*}$ \\
PTH-i &, 161 & $0,031^{*}$ \\
Cálcio &,- 035 & 0,612 \\
Fósforo &, 081 & 0,236 \\
\hline
\end{tabular}

$* p<0,05$ 
pacientes com DRC de moderada a leve (a maioria dos quais apresentavam idade $>60$ anos).

Nesse trabalho, os autores encontraram, aproximadamente, uma probabilidade duas vezes maior de ser frágil quando compararam pacientes com DRC e indivíduos com função renal normal. Já nos pacientes com DRC em TRS, a fragilidade, além de ser extraordinariamente mais prevalente (chegando a atingir $68 \%$ ), foi caracterizada em pacientes não idosos. No nosso estudo, $38,4 \%$ dos pacientes com DRC eram frágeis, com prevalência igualmente distribuída entre as modalidades de tratamento conservador, hemodialítico e diálise peritoneal. Confirmamos também que o construto de fragilidade ocorre em pacientes não idosos com DRC e em TRS. Uma contribuição nova do nosso estudo foi a observação da ocorrência de fragilidade em pacientes não idosos com DRC e em tratamento conservador. Portanto, a falta de uma interseção entre a idade e a fragilidade sugere que a fragilidade ou seus componentes são relevantes entre pacientes com DRC, sejam eles idosos ou não.

Até o momento, não dispomos de dados publicados sobre fragilidade na população brasileira. No estudo de Alvarado et al. ${ }^{13}$, realizado em pacientes idosos de ambos os sexos em sete cidades da América Latina e Caribe, a prevalência de fragilidade encontrada em São Paulo (que contribui com 2.143 participantes) foi $40,6 \%$. Contudo, o estudo se restringiu a cidade de São Paulo e, como não disponibiliza detalhamento dos dados clínico-laboratoriais dos participantes, não permite inferir a ocorrência de doenças e comorbidades presentes. A publicação dos dados completos do estudo Fragilidade em Idosos Brasileiros (FIBRA) possivelmente estabelecerá a prevalência da fragilidade na população idosa brasileira.

Em seu trabalho, Shlipak et al. ${ }^{8}$ observaram que a maior probabilidade de fragilidade em pacientes idosos com DRC comparativamente aos participantes com função renal normal permaneceu estatisticamente diferente após múltiplos ajustes para variáveis confundidoras, entre elas a raça e o sexo. Os autores destacam a maior frequência do diagnóstico de fragilidade particularmente entre as mulheres de cor negra (34\%), o que diferenciou de nossos achados de síndrome frágil menos frequente nas mulheres de raça negra, em todo espectro da DRC.

No nosso estudo, foi observada uma alta frequência dos componentes clínicos da síndrome frágil em todos os grupos de pacientes estudados, com exceção da perda não intencional de peso, que foi menos frequente, porém mais prevalente nos pacientes submetidos à DP. Chamam a atenção os altos percentuais de inatividade física (estatisticamente mais frequente entre os pacientes hemodialisados) pela sua importância na manutenção da capacidade funcional, na redução da sarcopenia, na melhoria do condicionamento cardiovascular e, por conseguinte, na prevenção e no tratamento das complicações e comorbidades observadas na DRC, causada principalmente pela hipertensão arterial e pelo diabetes mellitus, como nos nossos pacientes.

Apesar da alta prevalência, nossos dados não nos permite determinar a associação entre a DRC e a fragilidade, particularmente entre os pacientes não idosos, embora a ocorrência frequente de alterações inflamatórias e metabólicas possa contribuir. De fato, mediadores de declínio funcional aumentado, como estresse oxidativo elevado, endocrinopatias (deficiência do hormônio do crescimento, testosterona, hipotireoidismo), altos níveis das citocinas inflamatórias interleucina- 6 e fator de necrose tumoral- $\alpha$, são comuns na DRC e têm sido relatados também na fragilidade.

Um achado comum na DRC é a presença de anemia, complicação que limita a atividade física, associada à fraqueza e determinante da fadiga, todos componentes da síndrome frágil. Em nossos dados, embora tenhamos observado uma relação inversa entre a fragilidade e o nível de hemoglobina, esta se encontrava na faixa recomendada pelo K/DOQI ${ }^{12} \mathrm{e}$ $\mathrm{SBN}^{14}$, não sendo observada correlação entre o uso de AEE e fragilidade.

Outra possibilidade seria a ocorrência de anorexia e de náusea determinando limitação da ingestão alimentar, perda de peso e sarcopenia. Embora a anorexia e a náusea pudessem explicar a maior ocorrência de fragilidade nos pacientes em HD e DP, a presença desses sintomas é incomum nos pacientes com Kt/V adequado e em TC, particularmente quando a TFG encontra-se acima de $15 \mathrm{~mL} / \mathrm{min} / 1,73 \mathrm{~m}^{2}$, como foi o caso dos nossos pacientes. Adicionalmente, o estado inflamatório observado em todo espectro da DRC pode contribuir para a perda do apetite e consequente desnutrição. Contudo, a observação de níveis de albumina na faixa recomendada nos pacientes em TC e HD nos permite afastar a desnutrição como causa de fragilidade nos pacientes avaliados.

Distúrbios metabólicos como a acidose metabólica e distúrbios mineral e ósseo são comuns na DRC e podem favorecer a ocorrência da fragilidade. Algumas das consequências adversas decorrentes da acidose metabólica, como perda acentuada da massa muscular, doença óssea, anormalidades na secreção do hormônio de crescimento e tireoideano, são particularmente importantes para tornar o paciente 
com DRC mais predisposto a desenvolver a síndrome frágil. Infelizmente, a indisponibilidade dos valores de bicarbonato sanguíneo em todos os pacientes estudados limitou avaliar o impacto da acidose metabólica na prevalência da fragilidade.

A relação entre os níveis inadequados de 25-hidroxivitamina $\mathrm{D}$ e fragilidade em sujeitos não portadores de DRC é conhecida. ${ }^{15,16}$ Níveis séricos insuficientes de 25-hidroxivitamina D têm sido associados ao aumento da dor, ${ }^{17}$ à piora da função muscular, ${ }^{18}$ a fraturas, ${ }^{19,20}$ à sarcopenia ${ }^{21}$ e é comum em pacientes com DRC. ${ }^{22}$ É sabido que a reposição de vitamina D, seja na sua forma ativa ou não, melhora as funções neuromuscular e física. ${ }^{23-26}$ Porém, até o momento, não existe estudo de intervenção que demonstre o efeito salutar da reposição de vitamina D na reversão da fragilidade.

Em nossa análise, observamos uma correlação inversa entre fazer uso de vitamina D (calcitriol ou colecalciferol) e fragilidade. Contudo, a falta de documentação clínica regular dos níveis de 25-hidroxivitamina $\mathrm{D}$ e/ou 1,25-dihidroxivitamina $\mathrm{D}$, particularmente nos pacientes em HD e DP, não nos permite afirmar se os pacientes com o fenótipo de fragilidade apresentavam níveis insuficientes de vitamina D.

Finalmente, é importante ressaltar a correlação inversa entre níveis de $\mathrm{PTH}$-i e fragilidade nos pacientes com DRC. O hiperparatireoidismo secundário é uma complicação frequente da DRC, particularmente nos estágios mais avançados da doença. O mecanismo pelo qual o hiperparatireoidismo se associa à fragilidade não pode ser estabelecido por meio do nosso estudo. A indisponibilidade de informações complementares associadas aos níveis séricos elevados de PTH-i e da dosagem de vitamina D nos limita a propor uma relação causal entre o hiperparatireoidismo e a fragilidade.

Nosso trabalho apresenta limitações. Primeiro, trata-se de um estudo transversal, que limita determinar se é a DRC que predispõe a fragilidade ou vice-versa. Segundo, os dados obtidos foram os disponíveis nas papeletas dos pacientes, o que limita a exploração da associação entre a fragilidade e a inflamação, o estresse oxidativo e outras alterações endocrinológicas e metabólicas comuns na DRC. Terceiro, trata-se de uma amostra composta com um número relativamente pequeno de pacientes e com representação amostral não uniforme entre os três grupos de tratamento estudados, acompanhados em um único centro. Finalmente, utilizamos um instrumento de avaliação da fragilidade ainda não validado no Brasil. Contudo, é importante ressaltar a não existência de tal instrumento em nosso meio, bem como a indisponibilidade de questionário considerado padrão ouro para a realização da validação. Por outro lado, três dos cinco pontos possíveis que compõem o instrumento utilizado são avaliados por meio de duas perguntas utilizadas no questionário de avaliação de qualidade de vida SF-36, ${ }^{27}$ já validado em nosso meio.

Descrevemos um grupo de pacientes em diferentes estágios da DRC e encontramos uma alta prevalência de fragilidade, independentemente da faixa etária. A fragilidade e seus componentes se associam com maior mortalidade e hospitalização em pacientes em TRS ${ }^{9}$ e em TC. ${ }^{10}$ Até o momento, os cuidados com a DRC têm sido focalizados na estabilização da TFG, diagnóstico e tratamento das complicações e comorbidades da doença, além da adequação do tratamento dialítico. A diminuição da TFG parece ser parte da queda progressiva do status funcional que predispõe a mortalidade aumentada. Se confirmada em outros estudos, a identificação da fragilidade em pacientes com DRC deveria receber intervenções especiais, objetivando preservar a independência, a qualidade de vida e a sobrevida dos pacientes.

\section{REFERÊNCIAS}

1. Obrador GT, Mahdavi-Mazdeh M, Collins AJ. On behalf of the Global Kidney Disease Prevention Network. Am J Kidney Dis 2011;57:361-70.

2. Wiggins J, Patel S. Management of chronic kidney disease in older adults. Aging Health 2010;6:41-51.

3. Bastos MG, Kirstajn GM. Doença renal crônica: importância do diagnóstico precoce, encaminhamento imediato e abordagem interdisciplinar estruturada para melhora do desfecho em pacientes ainda não submetidos à diálise. J Bras Nefrol 2011;33:93-108.

4. Kurella Tamura M, Covinsky KE, Chertow GM, Yaffe $\mathrm{K}$, Landefeld CS, McCulloch CE. Functional status of elderly adults before and after initiation of dialysis. $\mathrm{N}$ Engl J Med 2009;361:1539-47.

5. Jassal SV, Chiu E, Hladunewich MA. Loss of independence in patients starting dialysis at 80 years of age or older. N Engl J Med 2009;361:1612-13.

6. Fried LP, Tangen CM, Walston J, et al. Frailty in older adults: Evidence for a phenotype. J Gerontol A Biol Sci Med Sci 2001;56A: M146-56.

7. Fried LP, Lee JS, Shilipak M, et al. Chronic kidney disease and functional limitation in older people: health, aging and body composition study. J Am Geriatr Soc 2006;54:750-6.

8. Shlipak MG, Stehman-Breen C, Fried LF, et al. The presence of frailty in elderly persons with chronic renal insufficiency. Am J Kidney Dis 2004;43:861-7.

9. Johansen LK, Chertow GM, Jin C, Kutner NG. Significance of frailty among dialysis Patients. J Am Soc Nephrol 2007;18:2960-7.

10. Wilhelm-Leen ER, Hall YN, Tamura MK, Chertow GM. Frailty and chronic kidney disease: the Third 
National Health and Nutrition Evaluation Survey. Am J Med 2009;7:664-71.

11. Levey AS, Bosch JP, Lewis JB, Greene T, Rogers N, Roth D. A more accurate accurate method to estimate glomerular filtration rate from serum creatinine: a new prediction equation. Modification of Diet in Renal Disease Study Group. Ann Inter Med 1999;130:461-70.

12. National Kidney Foudation. K/DOQI clinical practice guidelines for chronic kidney disease: evaluation, classification and stratification. Am J Kidney Dis 2002; 39:S1-246.

13. Alvarado BE, Zunzunegui M-V, Béland F, Bamvita J-M. Life Course Social and Health Conditions Linked to Frailty in Latin American Older Men and Women. J Gerontol Med Sci 2008;63:1399-406.

14. Diretriz para o Tratamento da Anemia no Paciente com Doença Renal Crônica - J Bras Nefrol 2007;29:24-6.

15. Ensrud KE, Ewing SK, Fredman S, et al. Circulating 25-hydroxyvitamin D levels and frailty status in older women. J Clin Endocrinol Metab 2010;95:5266-73.

16. Shardell M, Hicks GE, Miller RR, et al. Association of low vitamin D levels with the frailty syndrome in men and women. J Gerontol A Biol Sci Med Sci 2009;64:69-75.

17. Heidari B, Shirvani JS, Firouzjahi A, Heidari P, HajianTilaki KO. Association between nonspecific skeletal pain and vitamin D deficiency. Int J Rheum Dis 2010;13:340-6.

18. Verhaar HJJ, Samson MM, Jansen PAF, de Vreede PL, Manten JW, Duursma SA. Muscle strength, functional mobility and vitamin D in older women. Aging 2000;12:455-60.

19. Holick MF. High prevalence of vitamin D inadequacy and implications for health. Mayo Clin Proc 2006;81:353-73.
20. Holick MF. The role of vitamin D for bone health and fracture prevention. Curr Osteoporos Rep 2006;4:96-102.

21. Visser M, Deeg DJH, Lips P. Low vitamin D and high parathyroid hormone levels as determinants of loss of muscle strength and muscle mass (sarcopenia): the Longitudinal Aging Study Amsterdam. J Clin Endocrinol Metab 2003;88:5766-72.

22. Cuppari L, Carvalho AB, Draibe SA. Vitamin D status of chronic kidney disease patients living in a sunny country. J Ren Nutri 2008;18:408-14.

23. Dhesi JK, Jackson SHD, Bearne LM, et al. Vitamin D supplementation improves neuromuscular function in older people who fall. Age and Ageing 2004;33:589-95.

24. Moreira-Pfrimer LDF, Pedrosa MAC, Teixeira B, Lazaretti-Castro M. Treatment of vitamin D deficiency increases lower limb muscle strength in institutionalized older people independently of regular physical activity: a randomized double-blind controlled trial. Ann Nutr Metab 2009;54:291-300.

25. Zhu K, Austin N, Devine A, Bruce D, Prince RL. A randomized controlled trial of the effects of vitamin $\mathrm{D}$ on muscle strength and mobility in older women with vitamin D insufficiency. J Am Geriatr Soc 2010;58:2063-8.

26. Ward KA, Das G, Roberts SA, et al. A randomized, controlled trial of vitamin D supplementation upon musculoskeletal health in post menarchal females. J Clin Endocrinol Metab 2010;95:4643-51.

27. Ciconelli RM, Ferraz, MB, Santos W. Tradução para a língua portuguesa e validação do questionário genérico de avaliação de qualidade de vida SF-36 (Brasil SF-36). Rev Bras de Reumatologia 1998;39:43-50. 